\title{
Early Visual Motion Experience Improves Retinal Encoding of Motion Directions
}

\author{
${ }^{\circledR}$ Li Zhang, ${ }^{1,2}{ }^{\circledR}$ Qiwen Wu, ${ }^{1,2}$ and ${ }^{\oplus}$ Yifeng Zhang ${ }^{1,3}$ \\ ${ }^{1}$ Institute of Neuroscience, Key Laboratory of Primate Neurobiology, Center for Excellence in Brain Science and Intelligence Technology, Chinese \\ Academy of Sciences, Shanghai 200031, ${ }^{2}$ University of Chinese Academy of Sciences, Beijing 100049, and ${ }^{3}$ Shanghai Center for Brain Science and \\ Brain-Inspired Intelligence Technology, Shanghai 201210, China
}

Altered sensory experience in early life often leads to altered response properties of the sensory neurons. This process is mostly thought to happen in the brain, not in the sensory organs. We show that in the mouse retina of both sexes, exposed to a motion-dominated visual environment from eye-opening, the ON-OFF direction selective ganglion cells (ooDSGCs) develop significantly stronger direction encoding ability for motion in all directions. This improvement occurs independent of the motion direction used for training. We demonstrated that this enhanced ability to encode motion direction is mainly attributed to increased response reliability of ooDSGCs. Closer examination revealed that the excitatory inputs from the ON bipolar pathway showed enhanced response reliability after the motion experience training, while other synaptic inputs remain relatively unchanged. Our results demonstrate that retina adapts to the visual environment during neonatal development.

Key words: direction selective ganglion cells; experience training; experience-dependent plasticity; motion encoding; retina

\section{Significance Statement}

We found that retina, as the first stage of visual sensation, can also be affected by experience dependent plasticity during development. Exposure to a motion enriched visual environment immediately after eye-opening greatly improves motion direction encoding by direction selective retinal ganglion cells (RGCs). These results motivate future studies aimed at understanding how visual experience shapes the retinal circuits and the response properties of retinal neurons.

\section{Introduction}

The nervous system is highly adaptive to the environment. During the critical period, when the nervous system is developed enough to process sensory inputs, while still retaining an elevated level of plasticity, sensory experience exerts a significant impact on its structure and function (Hensch, 2005; Levelt and Hübener, 2012; Rose and Bonhoeffer, 2018). Most notably, many aspects of the sensory system development can be shaped by early sensory experience, such as the emergence of ocular dominance (Hubel and Wiesel, 1970; Smith and Trachtenberg, 2007), the development of direction selectivity (Li et al., 2008) and orientation selectivity (Sengpiel et al., 1999) in the visual cortex, the formation of tonotopic map in

\footnotetext{
Received Mar. 10, 2020; revised May 14, 2020; accepted May 16, 2020.

Author contributions: L.Z. and Y.Z. designed research; L.Z. and Q.W. performed research; L.Z., Q.W., and Y.Z. analyzed data; Y.Z. wrote the paper.

This work was supported by The Recruitment Program of Global Experts and Project supported by Shanghai Municipal Science and Technology Major Project Grant 2018SHZDZX05. We thank Ying Fang and Long Yang for help on MEA recording and Jian Zhao, Qingyi Li, and Wenting Huang for technical assistance.

The authors declare no competing financial interests.

Correspondence should be addressed to Yifeng Zhang at zhangyifeng@ion.ac.cn.

https://doi.org/10.1523/JNEUROSCI.0569-20.2020

Copyright $\odot 2020$ the authors
}

the auditory cortex (Zhang et al., 2001), and the organization of the glomerulus microcircuits in the olfactory bulb (Liu et al., 2016). Through this process, the nervous system ensures that it makes full use of the available neurons to best represent the animal's rearing environment.

Two effective approaches have been engaged to investigate the effect of visual experience on the development of the visual system, visual deprivation and altered visual experience. Visual deprivation is used to distinguish aspects of development that requires visual experience from other concurrent processes (Wiesel and Hubel, 1963; Borges and Berry, 1978; Melamed et al., 1986; Crair et al., 1998; Tian and Copenhagen, 2001; Dunn et al., 2013; Bos et al., 2016). Altered experience helps to identify visual functions that may not require early experience to develop initially but will nevertheless be shaped by early experience. Such adaptive changes, if occurred during the critical period, are often long term and cannot be reversed by visual experiences outside of that period (Blakemore and Cooper, 1970; Hirsch and Spinelli, 1970). Whereas visual deprivation, in many cases, halts the progress of development but often recover after normal visual experience is restored (Borges and Berry, 1978; Melamed et al., 1986; Bos et al., 2016). 
A

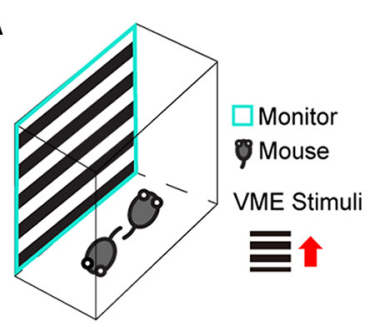

D

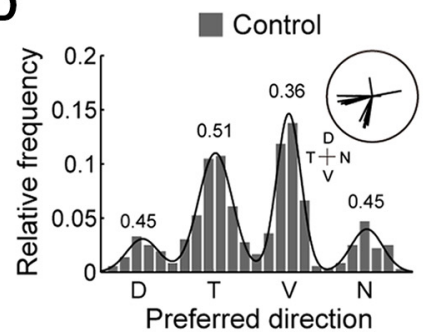

B

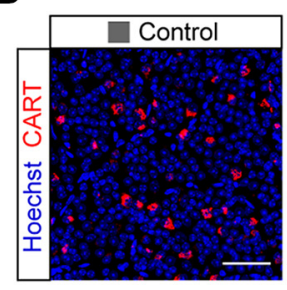

C

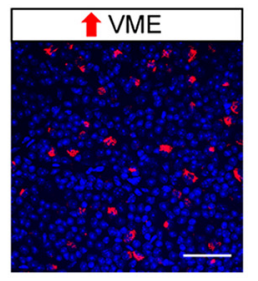

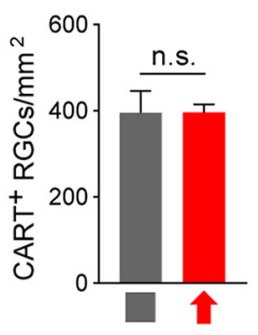

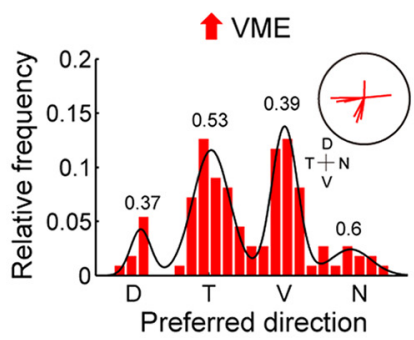

E

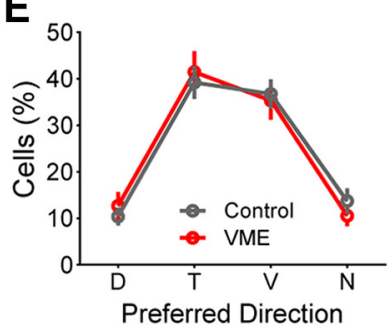

Figure 1. VME does not affect motion representation in the retina. $\boldsymbol{A}$, VME experimental design. $\boldsymbol{B}$, Example face-on images showing the expression of CART in the ganglion cell layers of the control and upward VME retinas. CART is a marker for most 00DSGCs. Scale bar, $50 \mu \mathrm{m}$. Each image is $240 \times 240 \mu \mathrm{m}$ in size and corresponds to $\sim 7^{\circ}$ in visual angle. $C$, Comparison of the average density of the CART-positive RGCs. Unpaired $t$ test, two-tailed, $p=0.98$, n.s. not statistically significant, for control, three FoVs (Field of view), one retina, for upward VME, six FoVs, two retinas. $\boldsymbol{D}$, Angular distribution of PDs for all 00DSGCs in the control (left) and VME (right) group. For each group, PDs of 0oDSGCs from an example piece are shown in the inset, PD distribution of all 00DSGCS recorded is shown with fitted Gaussian distribution (solid black line). SD (standard deviation) of each subtype of ooDSGCs was indicated individually above the fitted peaks. As a comparison, upward VME group has roughly the same distribution of PDs as the control group. D, dorsal; V, ventral; T, temporal; N, nasal. $\boldsymbol{E}$, Percentages of D/N/T/N subtypes in the 00DSGC population. Control, 28 pieces, D/N/T/N, 10.38/39.2/36.73/13.69\%; VME, 13 pieces, 12.68/41.46/35.33/10.53\%; multiple $t$ test, D/N/T/N, $p=5.00 \mathrm{E}-01 / 6.90 \mathrm{E}-01 / 7.90 \mathrm{E}-01 / 4.10 \mathrm{E}-01$. All error bars: SEM.

These studies have provided important insights on the development of the visual system. However, studies using altered visual experience have mostly focused on the primary visual cortex, and more recently, lateral geniculate nucleus (Chalupa and Rhoades, 1978; Engert et al., 2002; Kreile et al., 2011; Jaepel et al., 2017), but not the retina (see, however, Daw and Wyatt, 1974). In this report, we examined whether the response of retinal neurons can be altered by a biased visual experience at eye opening. The ON-OFF direction selective ganglion cell (ooDSGC) is the most well-studied type of retinal output neuron. They have known molecular markers (Huberman et al., 2009; Kay et al., 2011; Rivlin-Etzion et al., 2011; Dhande et al., 2013) and clearly defined trigger feature, a certain direction of motion (Barlow et al., 1964; Huberman et al., 2009; Kim et al., 2010). There are four subtypes with different motion direction preferences (Oyster and Barlow, 1967; Elstrott et al., 2008) but otherwise similar circuit arrangements (Demb, 2007; Briggman et al., 2011). The circuits are well established at postnatal day (P)12 and ooDSGCs are already directionally selective by eye opening ( $\sim$ P14; Elstrott et al., 2008; Wei et al., 2011). Visual deprivation around and after eye opening delays the realignment of preferred directions (PDs) but has no effect on the strength of direction selectivity of ooDSGCs (Chen et al., 2009; Bos et al., 2016). Nevertheless, a short visual adaptation in adult animals may induce a temporary reversal of PDs in ooDSGCs, indicating a certain degree of flexibility in the direction selective circuits (Rivlin-Etzion et al., 2012). These characteristics make ooDSGCs a particularly good subject of study in the exploration of visual experience dependent changes in the retina during early development.

In this report, we examine the effect of a biased early visual experience on the response properties of retinal ganglion cells (RGCs). Since ooDSGCs are important for motion encoding, we asked whether response properties of ooDSGCs may be affected by early visual experience enriched in motion. We also explored the potential mechanisms underlying the changes.

\section{Materials and Methods}

Experimental design and statistical analysis

All the experiments were designed with proper controls. Experimental design is described below in sections Visual motion training and Motion direction decoding. Student's unpaired $t$ test was used to compare differences between two groups. One-way ANOVA was used to compare multiple groups followed by a Bonferroni's multiple comparisons test. Two-way ANOVA followed by a Bonferroni's multiple comparisons test was used to compare visual motion experience (VME) training groups with control groups under different speeds/contrasts conditions.

\section{Mice}

C57BL/6 mice of either sex and from mixed litters were used in each experiment. Sample sizes (number of retinas and/or cells) are indicated in the figures or figure legends. All experimental procedures were approved by the Institutional Animal Care and Use Committee of the Institute of Neuroscience, Chinese Academy of Sciences.

\section{Visual motion training}

The home made "visual experience" cages are $50 \times 25 \times 40 \mathrm{~cm}$ in size and light proof. One wall of the cage $(50 \times 40 \mathrm{~cm})$ was covered by a computer monitor to provide the VME for $12 \mathrm{~h}$ each day from P10 to P35. For moving dots stimuli, 300 square dots with random combinations of intensities ( $\mathrm{I}=0-1$, see below for corresponding photon flux values), sizes $\left(0.3-128^{\circ}\right.$ in visual angle), and speeds $(29-310 \mathrm{~mm} / \mathrm{s}$ on screen, angular speed varies depending on the distance to the screen), travel across the screen either in the same direction, or in two opposite directions (Fig. 1A). Every $6 \mathrm{~min}$, a different set of 300 dots replaces the previous set, but the direction of motion remains the same. For stationary stimulus, random screen captures of the moving dot stimulus was presented on screen with a refresh rate of once every $30 \mathrm{~s}$. Hence, the stimulus has the same spatial and contrast patterns as the moving dot stimuli, but no motion. For moving gratings stimuli, square wave gratings with random combinations of contrasts $(40-100 \%)$, spatial periods $\left(1.4-157^{\circ}\right.$ bar width), and speeds $(12-120 \mathrm{~mm} / \mathrm{s}$ on screen, angular speed varies depending on the distance to the screen) travel across the screen either in the same direction or in two opposite directions. Every 5-30 s, a different gratings pattern replaces the previous pattern, but the direction of 
motion remains the same. The average intensity of the monitor $(I=0.5)$ was equivalent to the following photon flux values for the three mouse photoreceptors, each expressed at the wavelength of peak sensitivity: rod, $4.75 \times 104$ photons $/ \mathrm{s} / \mu \mathrm{m}^{2}$ at $500 \mathrm{~nm}$; M cone, $5.56 \times 104$ photons/ $\mathrm{s} / \mu \mathrm{m}^{2}$ at $511 \mathrm{~nm}$; $\mathrm{S}$ cone, $1.05 \times 103$ photons $/ \mathrm{s} / \mu \mathrm{m}^{2}$ at $370 \mathrm{~nm}$.

Tissue preparation and MEA recording

Mice were dark adapted for at least $2 \mathrm{~h}$. After dark adaptation, they were anesthetized using isoflurane, and subsequently euthanized by cervical dislocation. Retina dissections were performed under infrared or dim red illumination at room temperature. The orientation of the retina was determined using landmarks of vessels on the choroid membrane (Wei et al., 2010). Isolated retinas were incubated with the Ringer's solution $\left(110 \mathrm{~mm} \mathrm{NaCl}, 1 \mathrm{~mm} \mathrm{CaCl}_{2}, 2.5 \mathrm{~mm} \mathrm{KCl}, 1.6 \mathrm{~mm} \mathrm{MgCl}_{2}, 22 \mathrm{~mm}\right.$ $\mathrm{NaHCO}_{3}$, and $10 \mathrm{~mm}$ D-glucose, aerated with $95 \% \mathrm{O}_{2}$ and $5 \% \mathrm{CO}_{2}$ ) in a light-tight container. For recording, retinas were cut into elongated quadrilaterals ( $\sim 1$ by $1.5 \mathrm{~mm})$ and put on to the multielectrode array (MEA; 256MEA30/8iR-ITO, MCS) with the ganglion cell side down. The retina piece was then adapted in the dark for at least 20 min until a steady state of spontaneous activity was achieved. Throughout the recording, the retina piece was kept at $28-32^{\circ} \mathrm{C}$, with a perfusion speed of 4- to 6-ml Ringer's solution/min. Extracellular spikes were recorded by the USB-MEA256-System (MCS) at a sampling rate of $10 \mathrm{kHz}$. Spike sorting was done offline by a customized program (courtesy of Ofer Mazor, with modifications). All other analysis was performed in MATLAB.

\section{Visual stimulation}

Visual stimuli were delivered from a computer driven LED computer monitor to the photoreceptor layer of the retina piece through a custommade lens system (frame rate $60 \mathrm{~Hz}$, magnification $8 \mu \mathrm{m} /$ pixel). Black and white stimuli were used, and the average intensity for all stimuli was equivalent to the following photon flux values for the three mouse photoreceptors, each expressed at the wavelength of peak sensitivity: rod, $3.16 \times 103$ photons $/ \mathrm{s} / \mu^{2}$ at $500 \mathrm{~nm} ; \mathrm{M}$ cone, $3.71 \times 103$ photons $/ \mathrm{s} / \mu \mathrm{m}^{2}$ at $511 \mathrm{~nm}$; $S$ cone, $6.99 \times 101$ photons $/ \mathrm{s} / \mu \mathrm{m}^{2}$ at $370 \mathrm{~nm}$. The speed series of motion stimuli are traveling gratings with a $19.2^{\circ}$ spatial period and $100 \%$ grating contrast. The contrast series of motion stimuli are traveling gratings with a $19.2^{\circ}$ spatial period and $24 \%$ motion speed.

\section{Identification of ooDSGCs}

Direction selectivity of all RGCs was measured using a full field squarewave gratings stimulus moving in eight directions (spatial frequency $19.2 \%$ cycle, speed $5-119 \%$ s, $5 \%-100 \%$ contrast). Direction selectivity index (DSI) values were computed as described previously (Taylor and Vaney, 2002). An RGC was classified to be an ooDSGC if it is an ONOFF cell, its PDs under low to medium speeds or medium to high contrast are within a $60^{\circ}$ range, and its DSI at $24^{\circ} / \mathrm{s}, 100 \%$ contrast exceeds 0.1 . Approximately $6-20$ ooDSGCs could be identified from each piece of retina. PDs of ooDSGCs from a single piece of retina always cluster nicely in the four cardinal directions. Total number of ooDSGCs recorded under each VME conditions and subsequently used for decoding analysis are as follows (mice/retina pieces/ooDSGCs). For Figure $2 A-F$, control 5/9/50, upward VME (VME) 5/8/55, stationary dots (stationary) $4 / 6 / 52$. For Figures $3,4 A-D, 5 C, G, H$, control 9/16/133, upward VME (VME) 11/19/209, upward VME recorded at P120 (VME P120) $2 / 3 / 78$. For Figures $2 G, H, 4 E, F, 5 D, I-L$, control 18/31/205, backward VME (B.VME) 13/13/157, forward VME (F.VME) 14/16/112, horizontal VME (H.VME) 5/12/86, adult VME (Ad.VME) 8/19/100, upward VME (VME) 6/14/71, downward VME 5/13/100.

\section{Distribution of PDs}

Each recorded ooDSGCs was assigned to a subtype manually according to its $\mathrm{PD}$ and the orientation of the retina piece. Because of variations on the placement of each retina piece on the array, the measured PDs of the same subtype of ooDSGCs from different pieces of retina do not always match. This was corrected by shifting the PDs of each retina piece, so that the centers of distribution for the four subtypes across all pieces are the same. The PDs from all retina pieces were then pooled. The distribution of the pooled PDs was fitted to the sum of four Gaussians, with evenly spaced centers.

\section{Average direction tuning curve (TC)}

Direction TC (from $-\pi$ to $\pi$ ) of each ooDSGC was shifted so that the $\mathrm{PD}$ was centered at 0 . Average TCs was computed for different subtypes of ooDSGCs. The TC change between VME and control groups did not differ significantly for different subtypes of ooDSGCs, thus the data were pooled and presented as average of all ooDSGCs.

\section{Motion direction decoding}

All recorded responses of ooDSGCs to motion stimuli are pooled. The bootstrap method was used to select random subsets from the pooled data for decoding. For each iteration, equal number of cells are chosen randomly from each of the four cardinal groups (DVTN), and a random trial response from each cell was used for decoding. This process was then repeated 200-1000 times for each direction of motion under each speed or contrast condition. Throughout the analysis, response is defined as the total number of spikes fired during a single trial. A trial is defined as the traveling of one period of the gratings in a particular direction, thus the duration of the trial = spatial period/speed. The spatial period of the gratings was fixed at $19^{\circ}$ for all recordings. For the contrast series, the speed of the motion was $24^{\circ} / \mathrm{s}$, and a single trial lasted $0.79 \mathrm{~s}$ under all conditions. For the speed series, depending on the speeds of motion, a single trial lasted from $0.16 \mathrm{~s}\left(128^{\circ} / \mathrm{s}\right)$ to $3.81 \mathrm{~s}\left(5^{\circ} / \mathrm{s}\right)$.

Two decoding strategies are used to evaluate the encoding of motion directions by an ooDSGC population of size $N$ (Salinas and Abbott, 1994). For the vector method, the estimated direction of motion is denoted by the vector sum $V$ by defining:

$$
V=\sum_{i=1}^{N}\left(r_{i} * P D_{i}\right),
$$

where $\mathrm{r}_{\mathrm{i}}$ is the single trial response of cell $i, P D_{i}$ is a unit length vector denoting the PD of cell $i, N$ is the population size.

For the modified least-squares estimator method of decoding, mean responses of each cell under different directions of motion and speeds (or contrasts) were obtained as the cells empirical response TC. Note directions and speeds are treated as independent variables. For instance, for experiments that are consisted of eight motion directions and seven speeds, the TC of an ooDSGC includes its responses under all $8 \times 7$ conditions. The direction and speed (or contrast) of the motion are determined by a least-squared fit of the observed responses of $\mathrm{N}$ cells to the expected responses (TC):

$$
\left(\sum_{i=1}^{N}\left(r_{i}-T C_{i}\right)^{2}=\text { minimum }\right),
$$

where $r_{i}$ is the single trial response of cell $i$, and $T C_{i}$ is the TC of cell $i$ as described above.

In both methods, neuronal response $r_{i}$ is normalized so that the mean response to the cell's PD of motion under optimal speed and contrast is 1 .

\section{Prediction error and accuracy}

Prediction error is defined as the absolute value of angle difference between the predicted and the actual directions of motion. Prediction accuracy is the \% of correct decisions when choosing from eight possible actual motion directions ( $45^{\circ}$ interval, see stimulus protocol) based on the direction decoding results.

\section{Response variability}

Coefficient of variation (CV) of each cell under a particular stimulus condition is a scalar value defined as the standard deviation of all trial responses divided by the mean response. See above for the definition of a trial response. Only responses from the same cell under the same stimulus conditions including motion direction, speed and contrast are used to compute $\mathrm{CV}$, so even the same cell have different $\mathrm{CV}$ values under 
different stimulus conditions. $\mathrm{CV}$ value is taken as an indication of response variability.

\section{Matching response amplitude}

To match response amplitudes of two datasets, $\mathrm{A}$ and $\mathrm{B}$, with $\mathrm{A}$ having lower mean firing rate and $\mathrm{B}$ having higher mean firing rate, one trial with the highest single trial firing rate was removed from $B$, then one trial with the lowest single trial firing rate was removed from $\mathrm{A}$, bringing the mean firing rates of $\mathrm{A}$ and $\mathrm{B}$ closer to each other. After each removal, mean firing rates of the remaining trials from $\mathrm{A}$ and $B$ were compared. These steps were iterated until A and B had statistically matching mean firing rates. Each stimulus condition (speed/ contrast/direction) was processed and firing rate matched separately.

\section{Switching response variability}

To switch the response variability between VME and control groups, ooDSGCs from the two groups are paired based on their response amplitude, so that VME cells with high firing rates are paired with control cells with high rates, and vice versa. The strategy is to form simulated responses using the mean firing rate from one ooDSGC in the pair, and the response variability from the other in the pair. Let $r_{i}$ be the response of a VME ooDSGC at ith trial, $v$ be the CV for $r, r_{i}^{\prime}$ be the response at ith trial of a control ooDSGC, $v^{\prime}$ be the CV for $r^{\prime}$. From this VME-control pair, the response of the simulated cell in the $\mathrm{FR}_{\mathrm{VME}}+\mathrm{CV}_{\text {ctrl }}$ group, $R_{i}$, is defined either by

$$
R_{i}=\bar{r}+\frac{\left(r_{i}-\bar{r}\right) v^{\prime}}{v}
$$

or

$$
R_{i}=\overline{\mathrm{r}^{\prime}}+\frac{\left(r_{i}-\bar{r}\right) \bar{r}}{\bar{r}} .
$$

In both cases, $\bar{R}=\bar{r}$, and CV for $R$ equals $v_{2}$. Both achieved roughly the same results. The number of simulated cells used for decoding in the $\mathrm{FR}_{\mathrm{VME}}+\mathrm{CV}_{\mathrm{Ctrl}}$ group equals the number of cells in the VME group. Similar procedures are used to generate the $\mathrm{FR}_{\mathrm{Ctrl}}+$ $\mathrm{CV}_{\text {VME }}$ group.

\section{Histology and microscopy}

Immunohistochemistry on whole mount retinas was performed as described previously (Kim et al., 2008). Confocal microscopy was used to visualize and quantify the number of stained cells. Antibodies and reagents used were as follows: rabbit anti-CART (1:2000; Phoenix Pharmaceuticals, catalog H-003-62), Alexa Fluor 594 AffiniPure donkey anti-rabbit IgG (1:1000; Jackson ImmunoResearch, catalog 711-585-152), Hoechst 33258, pentahydrate (1:10,000; Invitrogen, catalog H3569).

\section{Intracellular recording}

Tissue preparation was the same as MEA recording. For functional identification of ooDSGCs, cell-attached recordings were per-

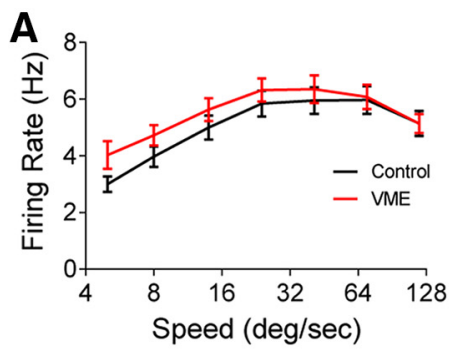

B
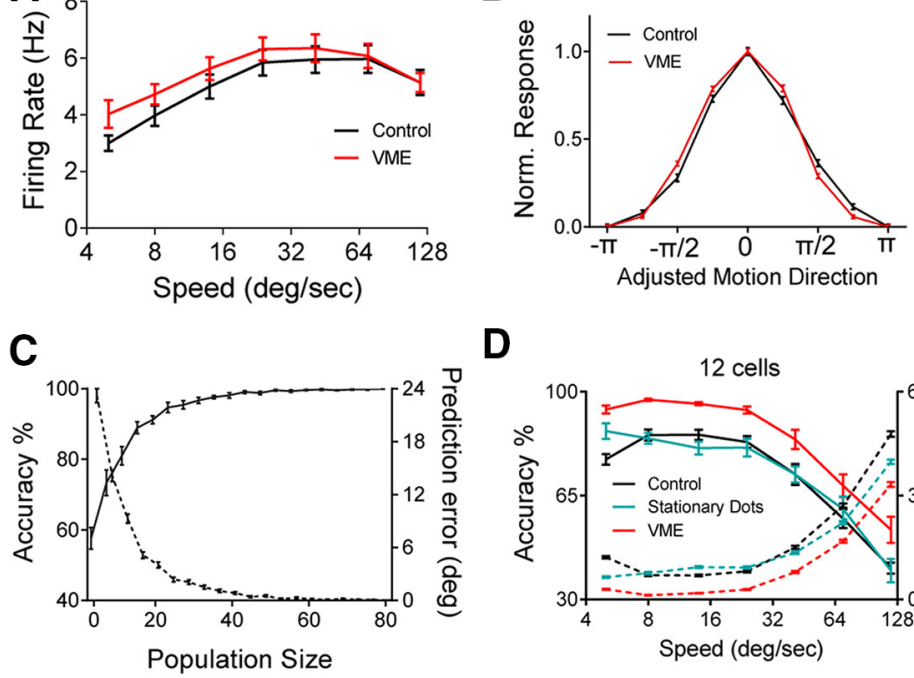

D
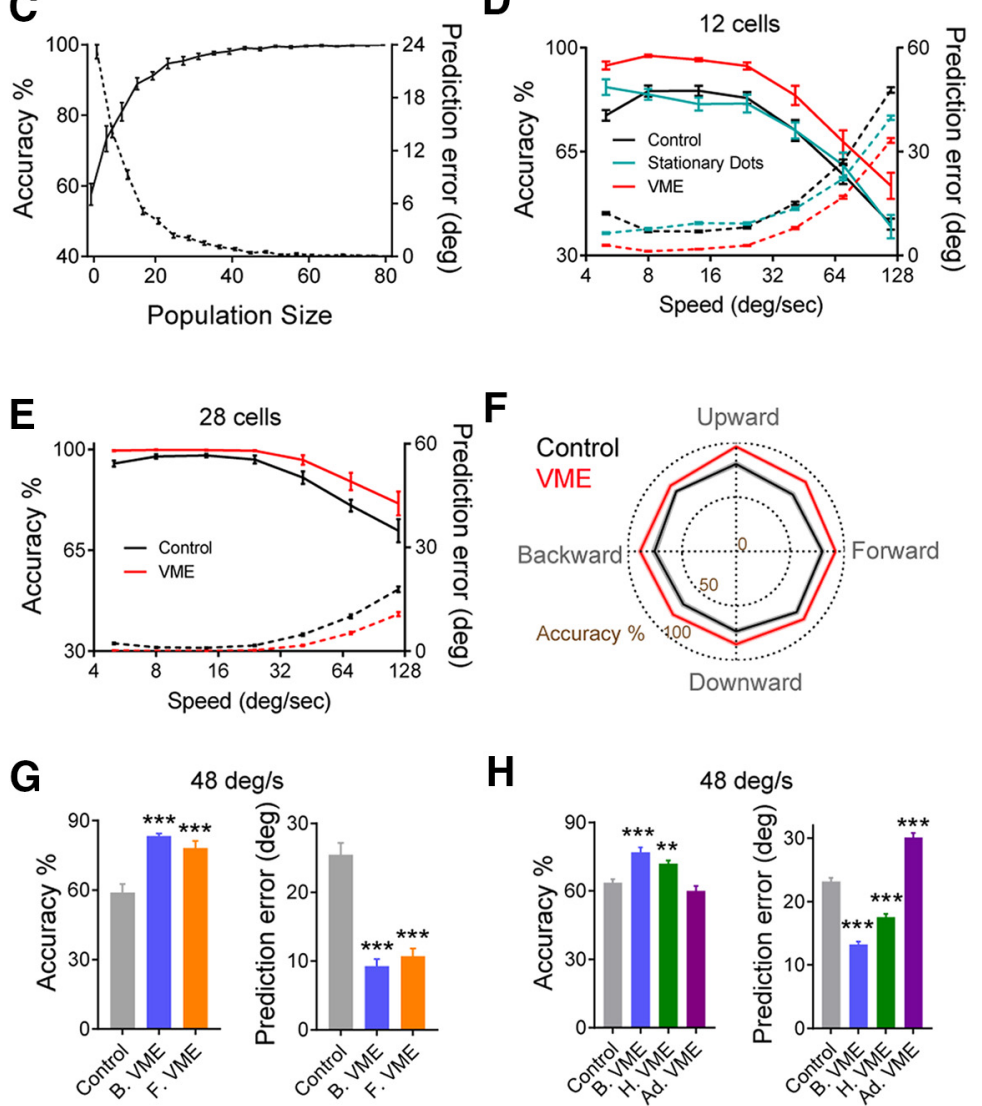

H

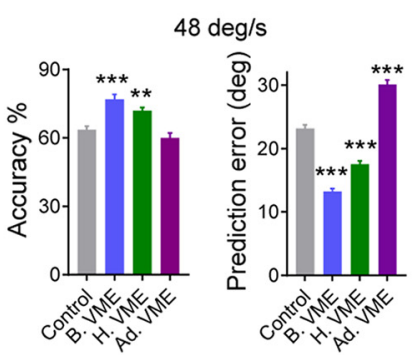

Figure 2. Decoding of motion directions is improved after early VME training. $A$, Average response of ooDSGCs to traveling gratings at different speeds. For each speed, responses to eight directions of motion are averaged. Control, 55 00DSGCS; VME, 50 ooDSGCS. $\boldsymbol{B}$, Average direction TCs of ooDSGCs in VME and control groups. Direction tuning of each ooDSGC was normalized so the response was between 0 and 1 before averaging. VME, 55 00DSGCs; control, 50 ooDSGCS. C, Direction decoding using 00DSGCs populations with varying sizes. Accuracy (solid line, left y-axis) and prediction error (dashed line, right $y$-axis) are both averaged from 1600 repeats. Each repeat uses randomly drawn single trial responses from a certain number of 0oDSGCS (population size, $x$-axis) to decode motion direction. Only data from control ooDSGCs are used here. $\boldsymbol{D}$, Comparison of direction decoding accuracy (solid lines, left $y$-axis) and direction prediction error (dashed lines, right $y$-axis) among the VME, stationary dots, and control groups. LSE method was used. Results are averaged from 4000 repeats, each using randomly selected single trial responses from 12 cells (three quartets of DVTN DSG(s). Two-way ANOVA with Bonferroni's multiple comparisons test, for accuracy: stationary dots versus control, $p=6.63 \mathrm{E}-01$, VME versus control, $p<1.00 \mathrm{E}-15$; for prediction errors: stationary dots versus control, $p<1.00 \mathrm{E}-15$, VME versus control, $p<1.00 \mathrm{E}-15$. $\boldsymbol{E}$, Same as $\boldsymbol{D}$ except the population size used for decoding is 28 cells (seven quartets of DVTN DSGCS). Comparing control and VME groups. $\boldsymbol{F}$, Decoding accuracy for different directions of motion. There is no overt direction tuning of accuracy in either the control or the VME group. Accuracy was obtained as described in $\boldsymbol{D}$ except the sample size was 500 for each direction. $\boldsymbol{G}$, Decoding backward motion using B.VME and F.VME groups. Decoding accuracy (left) and error (right) was obtained as described in D. One-way ANOVA with Bonferroni's multiple comparisons test, for B.VME, $p=2.46 \mathrm{E}-9$ (accuracy), $p<1.00 \mathrm{E}-15$ (error); for F.VME, $p=3.82 \mathrm{E}-06$ (accuracy), $p=2.50 \mathrm{E}-14$ (error), all compared with control. $\boldsymbol{H}$, Comparison of direction decoding accuracy (left) and error (right) after different types of VME training. Coding results for eight directions of motion are averaged. Ad.VME, VME training at adult age. One-way ANOVA with Bonferroni's multiple comparisons test, for B.VME, $p=1.39 \mathrm{E}-07$ (accuracy), $p<1.00 \mathrm{E}-15$ (error); for H.VME, $p=1.92 \mathrm{E}-03$ (accuracy), $p=4.00 \mathrm{E}-14$ (error); for Ad.VME, $p=4.49 \mathrm{E}-01$ (accuracy), $p<1.00 \mathrm{E}-15$ (error); all compared with control. ${ }^{* *} p<0.001$; ${ }^{* *} p<0.01$. All error bars: SEM. Error bars for prediction error in $\mathbf{C}-\mathbf{E}$ are too small to be noticed. 
A

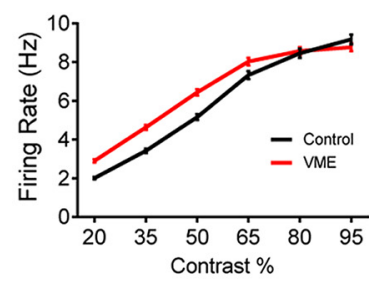

D

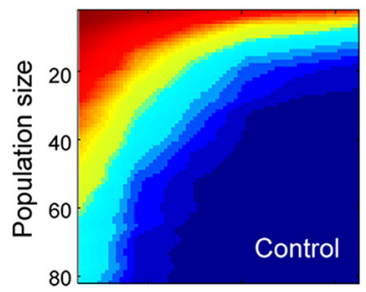

B

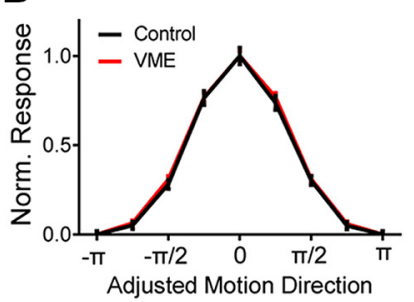

rror (deg)

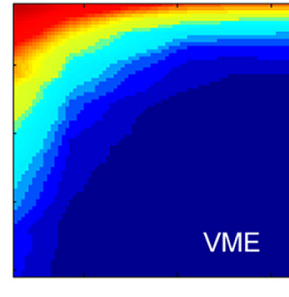

Accuracy \%

$\mathbf{E}$
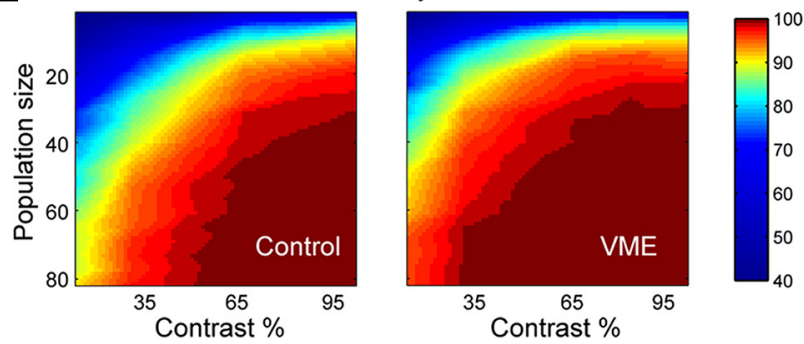

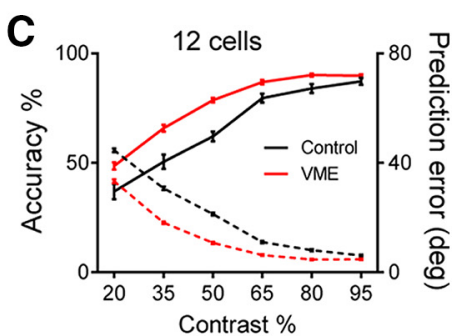

$\mathbf{F}$

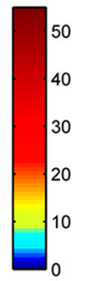

G

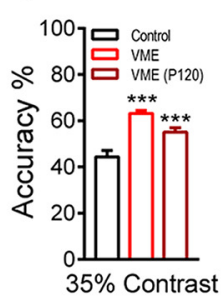

$35 \%$ Contrast
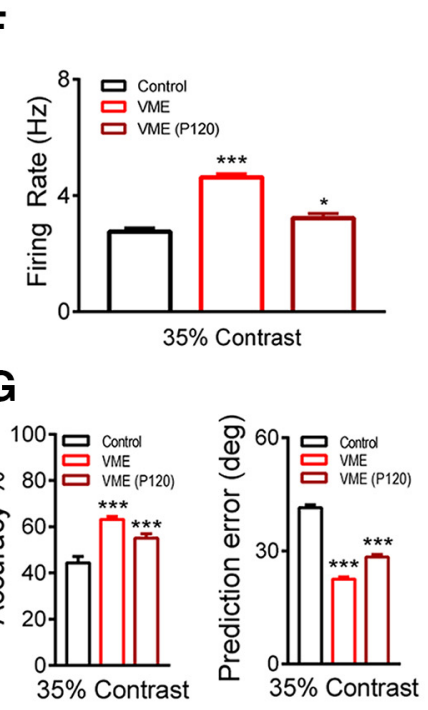

Figure 3. Direction encoding improves at different contrast levels and the improvement is long term. $\boldsymbol{A}$, Contrast tuning between VME and control (control, 133 00DSGCS, 16 retinas; VME, 209 00DSGCS, 19 retinas). B, Normalized direction TCs of ooDSGCs in VME and control groups. No significant change was detected. Control, 363 0oDSGCs; VME, 254 00DSGCS. C, Direction prediction error (dashed line, right $y$-axis) and accuracy (solid line, left $y$-axis) by the VME and control groups. LSE method was used. Each point represents average accuracy/error from 4000 repeats, each using randomly drawn single trial responses from 12 cells (three quartets of DVTN DSGCs). Two-way ANOVA with Bonferroni's multiple comparisons test, for accuracy: $p<1.00 \mathrm{E}-15$; for prediction error: $p<1.00 \mathrm{E}-15 . \boldsymbol{D}, \boldsymbol{E}$, Motion decoding performance $(\boldsymbol{F}$, prediction error; $\boldsymbol{G}$, accuracy) in relation to contrast and population size. Image was interpolated at intervals of $1 \%$ contrast and population size 1. VME shows better performance with higher prediction accuracy and smaller prediction error than control across a large range of contrast and the size of the decoding population. $\boldsymbol{F}$, Comparison of response amplitudes at 35\% contrast for different ages of VME groups with the control group. Control, 133 00DSGCs; VME, 209 0oDSGCs; VME (P120), 78 ooDSGCS. Unpaired $t$ test, for VME, $p<1.00 \mathrm{E}-15$; for VME (P120), $p=2.76 \mathrm{E}-04$, both compared with control. G, Direction decoding using 00DSGCs trained by early VME but recorded at different ages. Left, Accuracy. Right, Prediction error; one-way ANOVA with Bonferroni's multiple comparisons test, for VME, $p=6.93 \mathrm{E}-10$ (accuracy), $p<1.00 \mathrm{E}-15$ (error); for VME (P120), $p=8.29 \mathrm{E}-4$ (accuracy), $p<1.00 \mathrm{E}-15$ (error), all compared with control. ${ }^{* * *} p<0.001 ;{ }^{*} p<0.05$. All error bars: SEM. Error bars for prediction error in $C$ are too small to be noticed.

formed using 5- to 8-M $\Omega$ electrodes filled with Ringer's solution. To study the presynaptic inputs to ooDSGCs, voltage clamp recording was performed using 3- to $5-\mathrm{M} \Omega$ electrodes filled with internal solution (120 mM Cs methanesulfonate, $0.5 \mathrm{~mm} \mathrm{CaCl}_{2}, 5 \mathrm{~mm} \mathrm{NaCl}, 5 \mathrm{~mm}$ EGTA, 10 mM HEPES, 4 mM ATP, $0.5 \mathrm{~mm}$ GTP, and $5 \mathrm{~mm}$ QX-314, $\mathrm{pH}$ adjusted to 7.2 with $\mathrm{CsOH}$ ). Data were acquired by a MultiClamp 700B amplifier (Molecular Devices), low pass filtered at $1 \mathrm{kHz}$ and digitized at $10 \mathrm{kHz}$.

\section{Results}

\section{Motion representation in the retina is not affected by early} visual training

VME was provided to the mice for $12 \mathrm{~h}$ each day during an important developmental period for the visual system (Hooks and Chen, 2007) from P10 to P35. The motion experience consisted of dots or gratings of various spatial frequencies, contrasts, and speeds, all moving in one fixed direction (Fig. 1A). Tests on RGC function were performed after P35. During early development, a striped visual environment induced an increase in the proportion of orientation selective $\mathrm{V} 1$ neurons for the particular orientation (Blakemore and Cooper, 1970; O'Hashi et al., 2007; Kreile et al., 2011). In ferret V1, exposure to a specific direction of motion right after eye opening can induce a rapid emergence of direction selective neurons for the experienced direction (Li et al., 2008).
We thus first examined whether the ooDSGCs in the retina undergo similar changes to better represent the direction of motion enriched in the visual environment.

CART is a molecular marker for all ooDSGCs (Kay et al., 2011). In the VME and the control retinas, CART expressing cells were at roughly the same density $\left(\sim 400\right.$ cells $/ \mathrm{mm}^{2}$; Fig. $1 B$, $C)$. MEA recordings of isolated retinas were performed. ooDSGCs were identified by their differential responses to square-wave gratings moving in eight different directions. The proportion of ooDSGCs in all recorded RGCs did not differ between the VME and the control groups (VME, 14.69\%; control, $14.41 \%, p=0.74$, Fisher's exact test). Hence, after VME training, the ooDSGCs did not undergo changes that affected their overall survival or identity.

The ooDSGCs can be divided into four subtypes (Elstrott et al., 2008; Sabbah et al., 2017; see also Fig. 1D, left inset), each preferring a different direction of motion: ventral, dorsal, temporal, and nasal direction on the retina (V-DSGC, D-DSGC, T-DSGC, and N-DSGC, respectively). The numbers of DVTN cells are roughly equal in the normal retina (Bos et al., 2016). However, in our MEA recording results, there are higher proportions of $\mathrm{V}$ DSGCs and T-DSGCs than the N-DSGCs and D-DSGCs in both the VME and the control retinas. Similar results have been 
A

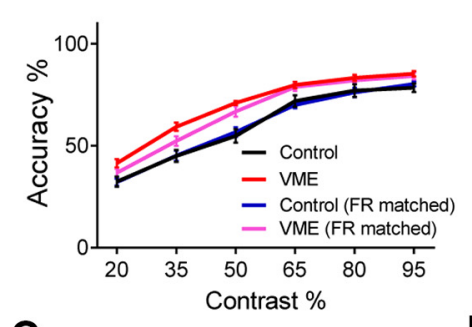

C

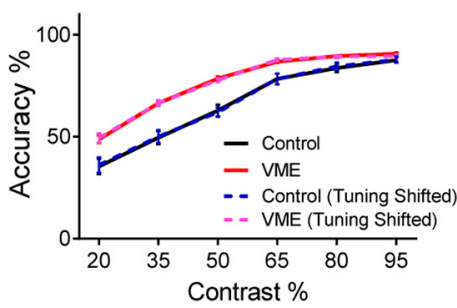

B

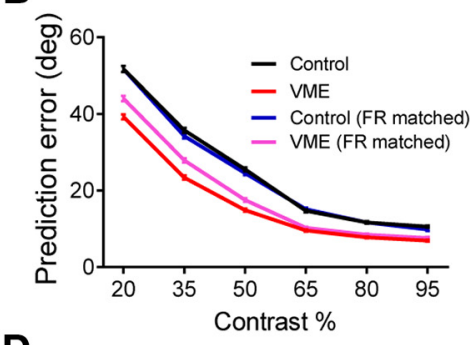

D

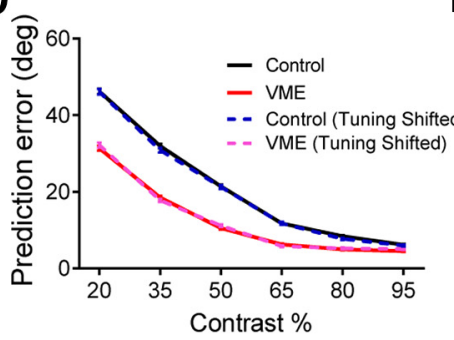

$\mathbf{E}$

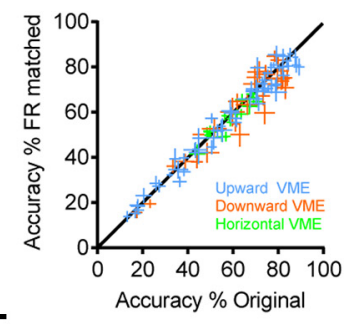

$\mathbf{F}$

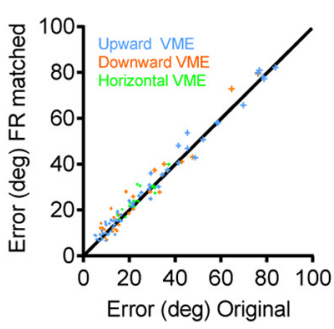

Figure 4. Firing rate does not affect motion encoding. $\boldsymbol{A}, \boldsymbol{B}$, Motion direction decoding accuracy $(\boldsymbol{A})$ and error $(\boldsymbol{B})$ using 0oDSGCs from the VME, control, and firing rate matched groups. There is no difference in performance between control (black) and firing rate matched control (blue). Between firing rate matched VME (magenta) and VME, there is no difference at higher contrasts, but some partial decrease in performance at lower contrasts. Two-way ANOVA with Bonferroni's multiple comparisons test, for accuracy in $A$, compare control and control (FR matched) groups, $p=9.25 \mathrm{E}-01$; compare VME and VME (FR matched) groups, $p=3.10 \mathrm{E}-03$; for error in $\boldsymbol{B}$, compare control and control (FR matched) groups, $p=1.07 \mathrm{E}-01$; compare VME and VME (FR matched) groups, $p<1.00 E-15$. C, D, Motion direction prediction accuracy $(\boldsymbol{C})$ and error $(\boldsymbol{D})$ by the VME, control, and tuning shifted groups. Shifting the tuning has no impact on the decoding of motion directions. Two-way ANOVA with Bonferroni's multiple comparisons test, for accuracy in $C$, compare control and control (tuning shifted) groups, $p=8.70 \mathrm{E}-01$; compare VME and VME (tuning shifted) groups, $p=8.83 \mathrm{E}-01$; for error in $\boldsymbol{D}$, compare control and control (tuning shifted) groups, $p=1.94 \mathrm{E}-01$; compare VME and VME (tuning shifted) groups, $p=4.17 \mathrm{E}-01$. $\boldsymbol{E}, \boldsymbol{F}$, Bulk comparison of direction decoding performance $(\boldsymbol{E}$, accuracy; $\boldsymbol{F}$, prediction error) before and after firing rate match. Each point in the graph represents coding performance of a group of VME ooDSGCS (color coded) at a given speed or contrast. The direction decoding accuracy and error using the entire dataset ( $x$-axis) or a subset of the data chosen to match the mean firing rate of the corresponding control group (y-axis) are compared. All points cluster around the identity line (black line), suggesting that the coding difference between VME and control groups is not caused by differences in their response amplitudes. All error bars: SEM. Error bars for prediction error in $\boldsymbol{B}, \boldsymbol{D}$, and $\boldsymbol{F}$ are too small to be noticed.

reported elsewhere and were suggested to be due to the sampling bias of the MEA method (Elstrott et al., 2008; Yonehara et al., 2016). Fortunately, this sampling bias is stable and consistent across all of our recordings. Therefore, comparison between the control and VME groups should be able to reveal qualitative changes in the proportions of $\mathrm{D} / \mathrm{V} / \mathrm{T} / \mathrm{N}$ subtypes. ON-OFF DSGCs trained by upward motion were analyzed (Fig. 1E). Since the lens reverses the image, upward motion is best detected by V-DSGCs. In the control and upward VME groups, the proportions of V-DSGCs were $36.7 \%$ and $35.3 \%$, respectively, not statistically different from each other ( $p=0.78$, unpaired $t$ test). This shows that early VME of upward motion did not alter the number of ooDSGCs that detect upward motion. Within each subtype of ooDSGCs, the PDs of ooDSGCs have a Gaussian like distribution (Fig. 1D). Visual deprivation from P3 to adult ( $>$ P30) delays the realignment of PDs so that they do not cluster as tightly into four groups as in normally reared animals (Bos et al., 2016). We tested whether VME has an impact on this realignment process. The standard deviation of the PDs indicates their spread, and the values for the V-DSGCs in the upward VME and control groups are 0.36 and 0.39 , respectively. The two distributions are not statistically different from each other $(p=0.52, F$ test). No difference was observed for the other three subtypes of ooDSGCs either $(p=0.12,0.26,0.88$ for D-DSGC/T-DSGC/NDSGC, $F$ test).

Overall, our results suggest that VME enriched in a single direction of motion did not affect the gross development of ooDSGCs or the distribution of their PDs, nor did it induce the retina to devote more ooDSGCs to the training direction. We conclude that the representation of motion by ooDSGCs is not affected by a biased VME training.

\section{Motion decoding from ooDSGC responses improves after early VME}

One major function ooDSGCs perform is the encoding of motion directions. We examined whether VME has an impact on the ability of ooDSGCs to encode motion directions. The motion stimuli used for VME training includes motion that span a wide range of speeds and contrasts. We first analyzed the responses of ooDSGCs to motion at different speeds. As has been observed previously (Nowak et al., 2011; Dhande et al., 2013), although the response amplitude is modulated considerably by motion speed (Fig. $2 A$ ), the shapes of the direction tuning remain relatively stable (Fig. $2 B$ ). This is true for both the control and the VME ooDSGCs. We then asked whether their ability to encode motion directions was affected by VME. To do this, we recorded ooDSGCs' responses to a traveling gratings stimulus that includes motion in eight different directions. We then examined how accurately we can identify the direction of motion out of eight possibilities using single trial responses of ooDSGCs. Two linear decoding strategies were employed: least squares estimator (LSE) that uses the direction TCs of each ooDSGCs, and the vector method that uses PDs of ooDSGCs (Salinas and Abbott, 1994). The same conclusions can be drawn using either decoding strategy, so only results from the LSE method are shown. Single trial responses of all recorded ooDSGCs to the same motion stimulus were combined and then subjected to bootstrap sampling. For each direction of motion, 200-500 samples, each contains random single trial responses from equal number of D-DSGC/V-DSGC/T-DSGC/N-DSGC, were used to decode motion direction. As can be expected, the larger the decoding population, the better the performance (Fig. $2 C)$. However, small moving objects can only excite a few 
A

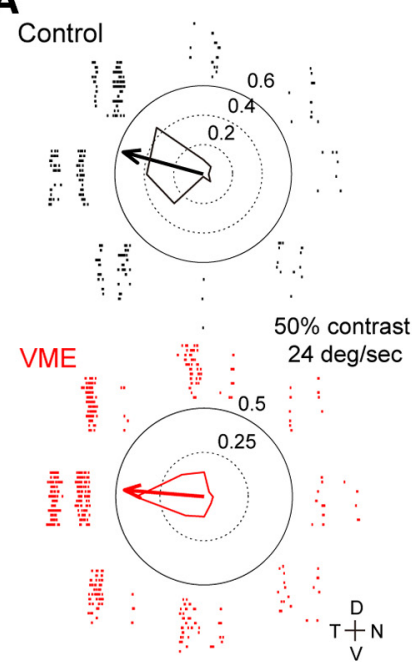

E

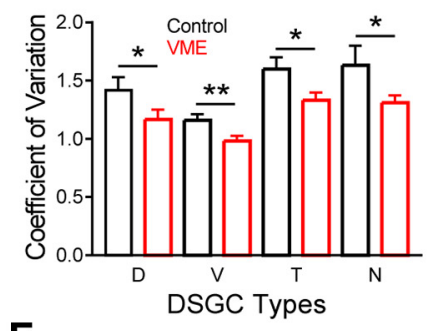

$\mathbf{F}$

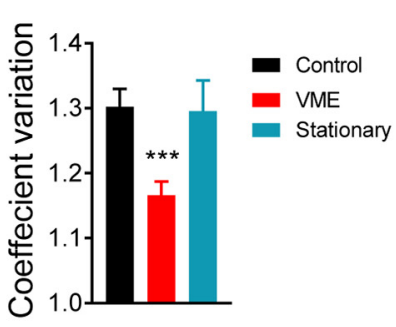

B

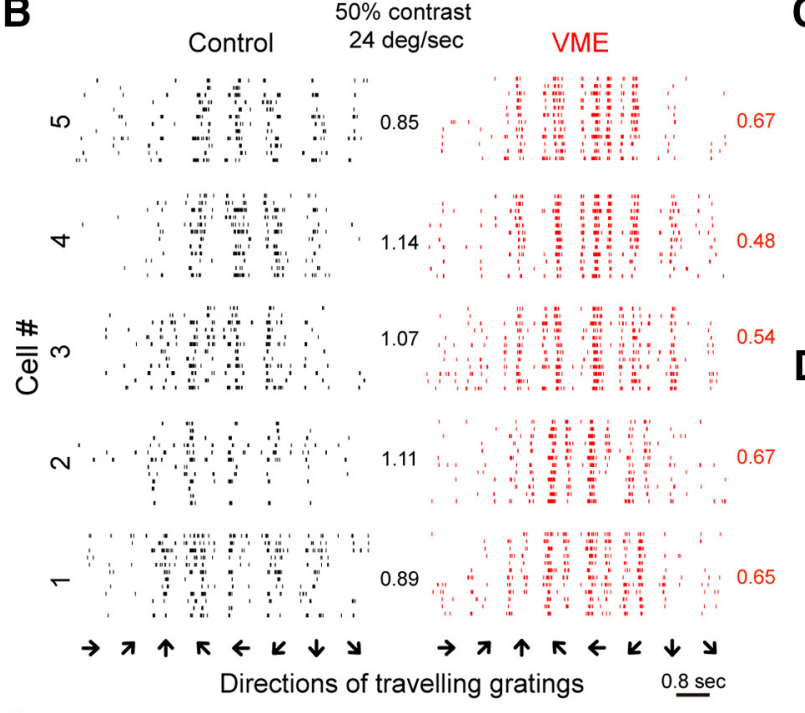

G

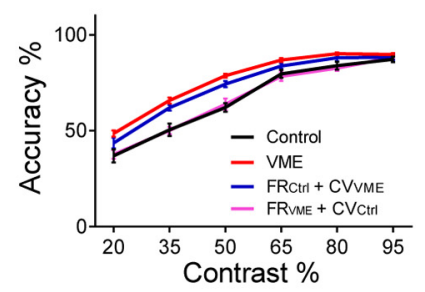

H

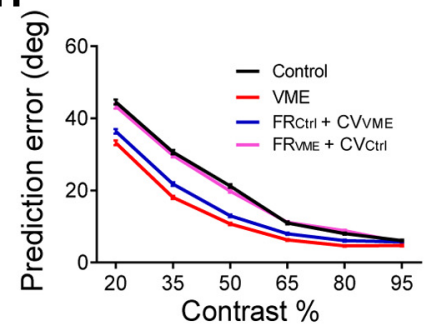

K
C

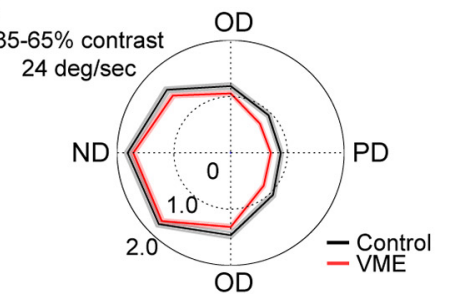

D

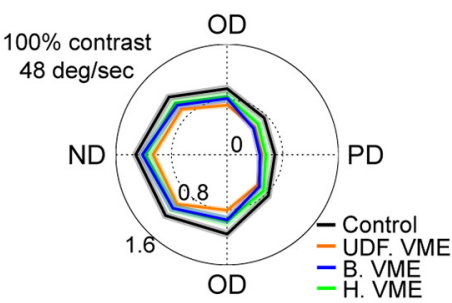

$\mathbf{J}$
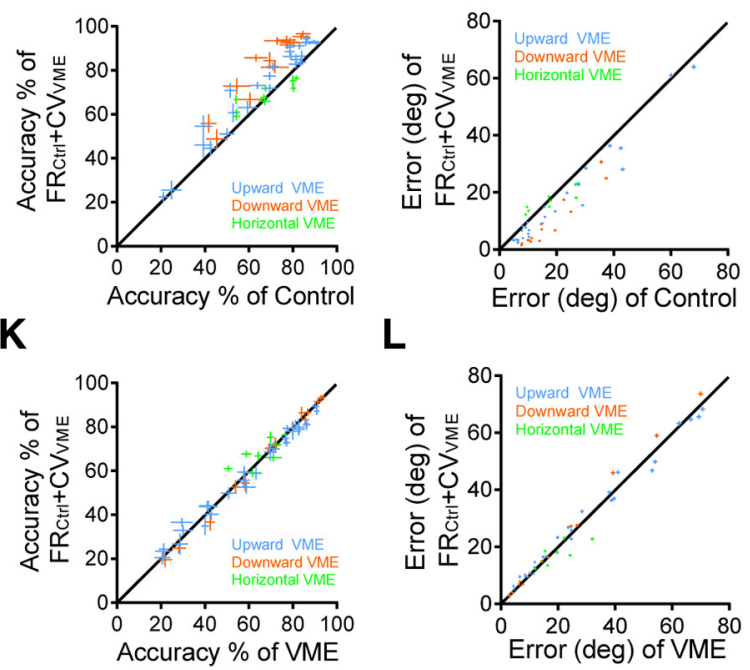

$\mathbf{-}$

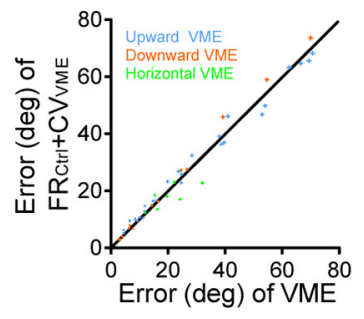

Figure 5. Increased response reliability underlies the improved motion encoding. $A$, Representative rasters and polar plots of two 0oDSGCs from the control (top) and VME (bottom) groups. The stimulus was traveling gratings in eight different directions. Polar plots show normalized responses with arrows indicating the PDs. $\boldsymbol{B}$, Representative rasters of five ooDSGCs each from the control (black) and VME (red) groups. The stimulus is the same as in A. Arrows at the bottom indicate the directions of motion. CV for each cell is indicated on the right. Only T-DSGCs are presented here for easier comparison across different cells. C, CV differs between VME and control. CV values are separately computed for PD/ND/OD directions and shown in the polar plot. For motion directions around and close to PD, VME O0DSGCS show significantly lower response variability as indicated by CV. OD, orthogonal directions. Two-way ANOVA with Bonferroni's multiple comparisons test, $p<1.00 E-15$. D, Comparison of $\mathrm{CV}$ between control and various VME groups. UDF.VME, upward/downward/forward VME groups combined. $E$, CV decrease occurs in all four 00DSGC subtypes. Unpaired $t$ test, for D/N/T/N, $p=3.00 \mathrm{E}-02 / 5.00 \mathrm{E}-03 / 1.00 \mathrm{E}-02 / 2.00 \mathrm{E}-02$. $\boldsymbol{F}, \mathrm{CV}$ do not change for stationary dots group. Unpaired $t$ test, stationary dots versus control, $p=9.13 \mathrm{E}-01$; VME versus control, $p=8.47 \mathrm{E}-05 ; n$ DSGCS for control/VME/stationary dots groups: 133/209/36. G, $\boldsymbol{H}$, Motion direction prediction accuracy $(\boldsymbol{G})$ and error $(\boldsymbol{H})$ of simulated responses with response variability switched between VME and control groups. The original control and VME groups are included for comparison. There is no difference between control (black) and $\mathrm{FR}_{\mathrm{VME}}+\mathrm{CV}_{\mathrm{Crrl}}$ (magenta). $\mathrm{FR}_{\mathrm{Ctrl}}+\mathrm{CV}_{\mathrm{VME}}$ (blue) is close to but does not fully reach the low level of prediction error observed in the VME, although there is no difference between the two in accuracy. Two-way ANOVA with Bonferroni's multiple comparisons test, for accuracy in $\mathbf{G}$, compare control and $\mathrm{FR}_{\mathrm{VME}}+\mathrm{CV}_{\mathrm{Ctrl}}$ groups, $p=9.13 \mathrm{E}-01$; compare $\mathrm{VME}$ and FR $\mathrm{R}_{\mathrm{Ctr}}+\mathrm{CV}_{\mathrm{VME}} \mathrm{groups}$, $p=2.46 \mathrm{E}-04$; for error in $\boldsymbol{H}$, compare control and $\mathrm{FR}_{\mathrm{VME}}+\mathrm{CV}_{\text {ctrr }}$ groups, $p=7.95 \mathrm{E}-02$; compare VME and $\mathrm{FR}_{\text {(trr }}+\mathrm{CV}_{\mathrm{VME}}$ groups, $p<1.00 \mathrm{E}-15$. $\boldsymbol{I}$, $\boldsymbol{J}$, Bulk comparison of accuracy $(\boldsymbol{I})$ and prediction error $(\boldsymbol{l})$ between control and $\mathrm{FR}_{\mathrm{ctrl}}+\mathrm{CV}_{\mathrm{VME}}$. $\mathrm{FR}_{\mathrm{ctrl}}+\mathrm{CV}_{\mathrm{VME}}$ shares all the response characteristics of the control group with the exception that the trial-trial variability is scaled to match one of the VME groups. Each point in the graph represents coding performance of a control ooDSGCs group at a given motion speed or contrast ( $x$-axis) or the coding performance of the corresponding group of simulated $\mathrm{FR}_{\mathrm{Ctrl}}+\mathrm{CV}_{\mathrm{VME}}$ responses (y-axis). $\boldsymbol{K}, \boldsymbol{L}$, Same as in $\boldsymbol{I}, \boldsymbol{J}$, except the comparison is between the original VME datasets ( $\mathrm{x}$-axis) and the simulated $\mathrm{FR}_{\mathrm{Crr}}+\mathrm{CV}_{\mathrm{VME}}$ dataset. All points cluster around the identity line, indicating the two datasets with identical CV but different firing rates encode motion directions equally well, and response variability accounts for most of the coding differences between the VME and the control groups. ${ }^{* * *} p<0.001 ;{ }^{* *} p<0.01 ;{ }^{*} p<0.05$. All error bars: SEM. Error bars for prediction error in $\boldsymbol{H}, \boldsymbol{J}$, and $\boldsymbol{L}$ are too small to be noticed.

ooDSGCs, yet they can still be detected by animals, thus decoding should work reasonably well by a small number of ooDSGCs. We present decoding results mostly using 12 ooDSGCs (three DVTN quartets). On the mouse retina, 12 neighboring ooDSGCs cover a visual field of roughly $8^{\circ}$ in size (Fig. $1 B$ ). In the control retina, decoding accuracy of motion direction from ooDSGC responses is speed dependent, with the best performance at the low to medium range of speed (Fig. 2D). The
ooDSGCs in the VME group trained by upward motion share a similar speed dependency. In addition, at a population size of 12 , VME outperformed the control group significantly, with average motion direction prediction error decreasing $5-15^{\circ}$ and prediction accuracy increasing $10-17 \%$ at all speeds tested (Fig. 2D). With more ooDSGCs, coding accuracy increases for both VME and control, but the difference between them diminishes (Fig. $2 D, E)$. When the population size reaches 28 , the difference in 
coding between VME and control becomes insignificant for most motion speeds below $24^{\circ} / \mathrm{s}$, although still significant for higher speeds (Fig. 2E).

\section{Improvement on direction encoding arises independent of the direction of training motion}

The VME stimulus used in training was in the upward direction, so we examined whether the improvement on direction decoding is restricted to upward motion. We compared the decoding results across different directions of motion. Surprisingly, motion decoding from the upward VME group shows no preference for upward motion, there is roughly the same degree of improvement for all eight directions of motion tested (Fig. 2F). Aside from upward motion, downward, forward, backward, horizontal, and vertical directions of motion were also used as the VME stimuli. We compared the decoding of backward motion using two VME groups trained by opposite directions of motion, the backward and the forward motion. The two groups performed equally well, and both were significantly better than the untrained control (Fig. 2G). We also used dots moving horizontally in two opposite directions instead of one fixed direction as the VME training stimulus. Roughly the same extent of improvement was observed (Fig. 2H). Thus, the motion does not have to be in one consistent direction for VME training to take effect on direction encoding of ooDSGCs.

One might ask what aspect of the VME training induced the change in ooDSGCs, is it the motion or the spatial patterning of the VME stimuli? To answer this, we raised a group of mice using a stationary stimulus, with the same random squares spatial patterning that changes to a different pattern every $30 \mathrm{~s}$. ooDSGCs after this stationary visual training encode motion directions no better than the control group (Fig. 2D), suggesting that an enriched visual environment in object spatial frequencies and contrasts is not sufficient to induce change in ooDSGCs. Therefore, motion in the VME training is likely responsible for the improved motion encoding by ooDSGCs.

Experience-dependent plasticity typically occurs during a sensitive period in development. We tested whether VME training also has a sensitive period. Nursing dams that were exposed to the same VME training as their pups were recorded and analyzed in the same way after the training. No improvement on direction decoding was observed using ooDSGC responses recorded from these mice (Fig. 2H). This suggests that VME training induced ooDSGC plasticity does have a sensitive period, which should overlap with the period we used for training (P10-P35) but does not extend into adulthood.

Taken together, our results demonstrate that VME training during early life significantly improved direction encoding by ooDSGCs. The improvement appears uniform across all directions of motion regardless of the patterns of motion stimuli used for training.

\section{Improvement on direction encoding is long term}

The improvement on motion encoding also spans a wide range of contrasts. We recorded ooDSGCs' response to a traveling grating stimulus under different contrasts after VME. Similar to speed, contrast modulates the response amplitude of ooDSGCs without affecting their direction tuning (Fig. 3A,B; Nowak et al., 2011). VME training did not affect the direction tuning of ooDSGCs but changed their contrast tuning property, so that at medium to low contrasts, their response was more robust (Fig. $3 A$ ). The improvement of direction encoding was also observed (Fig. 3C): at all but the highest contrast levels, decoding using
VME ooDSGCs achieved significantly better accuracy than using the control ooDSGCs. Moreover, at all contrast levels including the highest one, VME ooDSGCs make smaller direction prediction errors than control. On average, there is a $37.2 \%$ decrease in direction prediction error in the VME group, whereas the change in firing rate is $18.5 \%$. With increasing number of cells used for decoding, the performance improves. But across a wide range of population size, and even at a population size of 80 , significant improvement can be observed in VME compared with control, especially for motion at lower contrasts (Fig. 3D,E). These results reveal that similar to speed, encoding of motion direction at different contrasts also improved significantly for the VME groups.

We then tested how long the effect on motion encoding persisted after the VME training ended. Mice were put back into normal housing condition and visual environment as all the control mice after exposed to upward VME from P10 to P35. Recordings were performed around P120. Response amplitude at lower contrasts remained higher than control, and significantly improved direction decoding performance was maintained (Fig. $3 F, G)$. These results demonstrate that the influence of early VME on motion encoding is long-term.

\section{Increased response reliability underlies the improved motion encoding}

Efficiency of direction encoding can be influenced by the shape and the width of the DS TCs, the distribution of the PDs in the ooDSGC population, modulation by non-directional parameters such as speed and contrast, and the response variability of each neuron (van Hateren, 1990; Nowak et al., 2011). Average direction TCs and the distribution of PDs did not change in the VME ooDSGCs (Figs. $1 D, 2 B, 3 B$ ). On the other hand, response amplitudes differ greatly, at different contrast and speed, between VME and control groups, as well as between different pieces of retina recorded (Figs. 2A, 3A, 4A). We first examined the effect of firing rate on the decoding performance. By selecting a subset of low firing responses from the high firing rate group, and a subset of high firing responses from the low firing rate group, we arbitrarily matched the mean firing rates between the VME and the control groups. Alternatively, actual responses from the control group were shifted to the same average level as the VME group by multiplying each trial response to a constant value. In both cases, the resulting data pools have matching contrast tuning of firing rates, but response statistics for each cell across different trials (other than the mean value) are maintained. In both cases, motion decoding using VME ooDSGCs still significantly outperforms decoding using the control ooDSGCs (Fig. 4A-D). We extended this analysis to other VME groups trained by different directions of motion, and ooDSGC responses recorded under different speeds and contrasts. Overall, simply changing or matching the firing rate of VME ooDSGCs to control, or vice versa, does not alter the direction encoding capability of the ooDSGC population, accuracy of decoding from their responses remains largely unchanged (Fig. $4 E, F)$. Thus, improved direction encoding in the VME ooDSGCs cannot be simply attributed to enhanced response amplitude of the population, instead, it is likely associated with the statistics among individual trial responses.

Response reliability is critical in any encoding scheme. Spiking responses of ooDSGCs to the traveling gratings stimulus in eight directions were recorded (Fig. 5A,B) and response reliability was analyzed. CV (SD divided by mean) has been used widely to characterize response reliability (Levine and Shefner, 1977; von Trapp et al., 2016). We examined whether average CV 
of ooDSGC response is changed by VME training. Response of each ooDSGC to motion in their PD, null direction (ND), and the orthogonal directions (OD; the two directions perpendicular to the PD-ND axis) were separately analyzed and compared. For $\mathrm{PD}$ and directions around $\mathrm{PD}$, a significant drop in average $\mathrm{CV}$ can be observed (Fig. 5C). This decrease in $\mathrm{CV}$, or increase in response reliability, is present in all VME groups examined, although the amount of change differs (Fig. 5D). Furthermore, the increase in response reliability occurred in all DVTN subtypes (Fig. $5 E$ ), consistent with the uniform coding enhancement for all directions of motion. This change is also motion training dependent, as ooDSGCs trained with stationary patterns show no such change in response reliability (Fig. $5 F$ ).

The rise in response reliability corresponds well with better motion encoding in the VME group, but is it sufficient to explain the full extent of the improvement, or do other factors also contribute? To examine this, we generated simulated responses that switched the response variability between the VME and control groups, using actual response characteristics from upward VME and control ooDSGCs. The simulated response of a "FR $\mathrm{VME}_{\mathrm{VME}}+$ $\mathrm{CV}_{\mathrm{Ctrl}}$ " ooDSGC has the same mean firing rate as a random ooDSGC from the VME group, but the response variability of a matching ooDSGC from the control group. Similarly, a simulated "FR $\mathrm{Ctrl}_{\mathrm{V}}+\mathrm{CV}_{\mathrm{VME}}$ " ooDSGC has the mean response of a control ooDSGC but the response variability of a VME ooDSGC. Since all other response characteristics, such as direction tuning and speed tuning of each ooDSGC, distribution of PDs in the population, are determined by mean response, the resulting two simulated datasets are basically the same as the VME and control groups, except that the response variability is switched between them. When these sets of responses were used for decoding, we found that switching response variability basically switched direction decoding performance: increasing the response variability of the VME response to the extent that its $\mathrm{CV}$ matches that of $\mathrm{CV}_{\mathrm{Ctrl}}$ was enough to lower the decoding performance to the level of the control group. Conversely, decreasing $\mathrm{CV}$ of the control group to match $\mathrm{CV}_{\mathrm{VME}}$ was sufficient to achieve the same performance as decoding from the VME group (Fig. 5G, $H$ ). Similar results were obtained using data from ooDSGCs trained by different VME motion or recorded under different motion stimuli. In all cases, lowering CV of control groups leads to increase in direction decoding accuracy and decrease in prediction error (Fig. 5I,J). Furthermore, overall, we see that datasets with matching $\mathrm{CV}$ also have matching direction decoding performance (Fig. $5 K, L$ ). Thus, the improvement of the VME group on motion direction encoding can be largely attributed to enhanced response reliability.

\section{On bipolar pathway becomes more reliable after VME training}

We next examined what changes may occur in the ooDSGC circuit to account for the effect brought about by VME training. We performed targeted voltage clamp recordings of ooDSGCs in the VME and control groups. Motion stimuli in both the preferred and NDs were presented, and EPSC and IPSC were recorded. Similar to previously reported, the ooDSGCs receive strong IPSC during motion in the ND, but much weaker IPSC in the PD, while the EPSCs are weaker in the ND than in the PD (Fig. $6 A)$. CV of peak IPSC and EPSC $\left(\mathrm{CV}_{\mathrm{E}}\right.$ and $\left.\mathrm{CV}_{\mathrm{I}}\right)$ was computed and compared (Fig. $6 B$ ). At $30 \%$ contrast, VME on average have $30.1 \%$ smaller $\mathrm{CV}_{\mathrm{E}}$ than control, the difference is statistically significant. At $100 \%$ contrast, there is also a $28.6 \%$ decrease in average $\mathrm{CV}_{\mathrm{E}}$ for the VME group, although the difference is
A

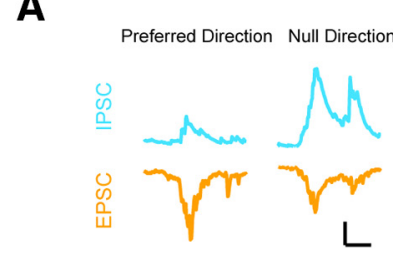

B

C

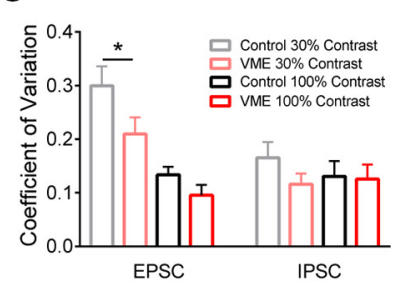

D

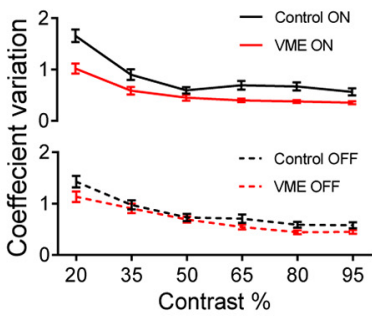

E

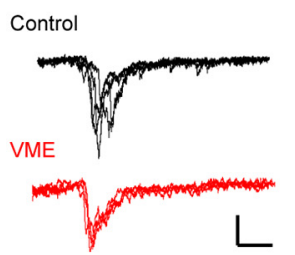

\section{G}

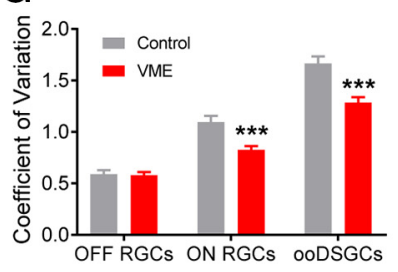

$\mathbf{F}$

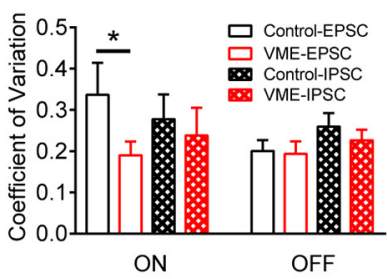

H

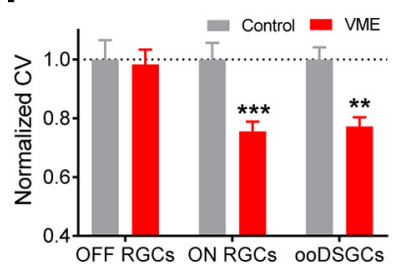

Figure 6. Excitatory inputs to ooDSGCS are more reliable after VME training. $A$, EPSC and IPSC of an ${ }_{00 D S G C}$ in response to traveling gratings in preferred and NDs. $\boldsymbol{B}$, Raw traces of EPSC (PD) and IPSC (ND) from two 00DSGCS in response to traveling gratings at $30 \%$ contrast. These are representative examples to illustrate more reliable VME EPSCS compared with control. C, Summary of response variability as measured by CV of peak EPSC and IPSCS at different stimulus contrasts. Traveling gratings in the PD (EPSC) and ND (IPSC) was used. Unpaired $t$ test, $p=3.73 \mathrm{E}-02$. Control, eight $00 \mathrm{DSGCS}$; VME, nine $00 \mathrm{DSGCS}$. D, Variability of ooDSGCS spiking responses to the onset and offset of a full field light flash at different contrasts. CV for ON (top) and OFF (bottom) responses are compared between upward VME and control groups. Two-way ANOVA with Bonferroni's multiple comparisons test, for ON (top), $p=1.19 \mathrm{E}-10$; for OFF (bottom), $p=2.43 \mathrm{E}-03$. Control, 73 00DSGCS; VME, 64. E, Raw traces of ON EPSC recorded from a control and a VME ooDSGC in response to flashes at 30\% contrast. These are representative examples to illustrate more reliable ON EPSCS for VME. $\boldsymbol{F}$, Summary of response variability as measured by CV of peak EPSC and IPSCS, with ON and OFF responses separately analyzed. The stimulus was full field flashes, data for 30\% and $100 \%$ contrasts are combined. Unpaired $t$ test, $p=4.83 \mathrm{E}-02$. Control, eight $00 \mathrm{DSGCS}$; VME, seven 00DSGCS. G, Comparison of response reliability among different RGC types. The stimulus used is traveling gratings at $120 \%$ and $100 \%$ contrast. ON-RGCs and 00DSGCS show lower $\mathrm{CV}$, hence higher response reliability in the VME group as compared with the control group. OFF-RGCs: $p=0.88$; control $n=77$, VME $n=72$; ON-RGCs: $p=3.99 \mathrm{E}-05$, control $n=74$, VME $n=101$; 00DSGCs: $p=9.86 \mathrm{E}-07$, control $n=73$, VME $n=52$. Unpaired $t$ test. $\boldsymbol{H}$, The same as $\mathbf{G}$ but using normalized CV. All CV values are normalized to the corresponding mean CV values of the control groups. OFF-RGCs: $p=0.80$; ON-RGCs: $p=1.55 \mathrm{E}-04$; 00DSGCs: $p=2.83 \mathrm{E}-03$. Unpaired $t$ test. The relative changes in $\mathrm{CV}$ are comparable between ON-RGC and 00DSGCS. ${ }^{* *} p<0.001 ;{ }^{* *} p<0.01 ;{ }^{*} p<0.05$. All error bars: SEM. Scale bars: horizontal, $250 \mathrm{~ms}$; vertical, $50 \mathrm{pA}$ (EPSC)/200 pA (IPSC).

not statistically significant. $\mathrm{CV}_{\mathrm{I}}$, on the other hand, are not significantly different between the two groups (Fig. 6C). This result suggests that the excitatory synaptic inputs from bipolar cells to ooDSGCs are more reliable after VME training, this improves 
the response reliability, and consequently the coding capabilities of ooDSGCs.

Both the ON and OFF bipolar cells provide excitatory inputs to ooDSGCs, we asked which one or whether both are involved in this VME induced change. To separately examine the $\mathrm{ON}$ and OFF pathways, an ON-OFF flashing stimulus was presented to ooDSGCs and their spiking responses analyzed. The average response variability of the ON response from VME ooDSGCs is markedly lower than control under most stimulus contrasts (Fig. $6 D$, top), while for the OFF response, the difference is not statistically significant, although there seems to be a tendency for VME to be lower than control (Fig. $6 D$, bottom). This suggests that changes induced by the VME training lie mostly in the ON bipolar pathway, although OFF pathway may also exert a weaker influence. Synaptic inputs during the ON-OFF flashing stimulus was then recorded and analyzed. The results corroborate the conclusions drawn from the spiking results. Changes in response variability is mostly restricted to the $\mathrm{ON}$ excitatory pathway: $\mathrm{CV}_{\mathrm{I}}$ is not notably affected by VME (Fig. $6 E, F$ ), $\mathrm{CV}_{\mathrm{E}}$ for $\mathrm{ON}$ response decreased $\sim 40 \%(p<0.05)$, while no significant change was observed for the OFF response (Fig. $6 F$ ).

If the ON excitatory pathways are affected by VME, other RGCs that receive inputs from them should also show a change in response reliability. We identified ON and OFF RGCs from MEA recordings based on their responses to full field flashes, then compared their response reliability to a motion stimulus. As expected, ON-RGCs after VME training have significantly lower $\mathrm{CV}$ values than the control group, while OFF-RGCs do not show this change (Fig. 6G). The relative changes in $\mathrm{CV}$ between ooDSGCs and ON-RGCs appear comparable (Fig. 6H), suggesting that at least a large portion of the ON-RGCs are affected.

Together, these results indicate that exposure to an enriched visual motion environment immediately after eye opening positively impacted the retinal circuit, mostly in the ON excitatory pathway, to enhance its response reliability and to a lesser extent, its response amplitude under low contrast. In ooDSGCs, this resulted in significantly improved ability to correctly encode motion directions.

\section{Discussion}

The main finding of this study is that an enriched visual environment, especially in moving objects of various contrasts and speeds, around the time of eye opening caused the ooDSGCs in the retina to undergo changes that led to more accurate direction coding. The changes can be mostly attributed to the synaptic inputs from the ON bipolar pathway becoming stronger and more reliable. We conclude that retina, like LGN and visual cortex, can also be permanently affected by early visual experience.

\section{Direction encoding and speed/contrast encoding}

ooDSGCs are thought to be involved in encoding motion directions (Barlow et al., 1964). Numerous decoding strategies involving directionally tuned firing rates and activity correlation have been used (Fiscella et al., 2015; Franke et al., 2016; Kühn and Gollisch, 2016, 2019; Zylberberg et al., 2016). In our current study, we used firing rates and simple linear methods to decode motion direction. For decoding motion directions, we randomly chose responses that are not necessarily recorded in the same trial, or even on the same piece of retina, thus influences from shared input, shared noise and synchronized activity among ooDSGCs are effectively excluded from our analysis. We expect this decoder to provide a lower bound on the encoding capacity of the ooDSGC population.

The responses of ooDSGCs are strongly affected by contrast and speed. In our dataset, there are no significant difference in the overall shape and width of the direction TCs under different contrast and speed conditions. This has been reported by others as well (Nowak et al., 2011); see, however, Yao et al. (2018). Furthermore, direction tuning also do not appear to be altered by visual motion training (Figs. $2 B, 3 B$ ). On the other hand, speed and contrast tuning of the response amplitude is somewhat changed in the VME ooDSGCs (Figs. $2 A, 3 A$ ), although there is great variability in the firing rates (control, 40 pieces of retina, $4.86 \pm 1.90 \mathrm{~Hz}$; VME, 39 pieces, $6.46 \pm 2.82 \mathrm{~Hz}$; mean $\pm \mathrm{SD}$ ). Simply from firing rates of ooDSGCs, the effects of speed and contrast cannot be separated. But as postulated previously, nondirectional motion parameters such speed, contrasts and luminance can be combined to form a "equivalent contrast" superparameter (Nowak et al., 2011). Our decoder can take into account the effect of such a superparameter on the TC amplitude, and outputs both motion direction and this superparameter. Since we only altered one non-directional parameter (speed/contrast) at a time, our decoder was able to give a prediction of speed or contrast. This decoder performed slightly better at determining motion direction than a decoder that only considers direction tuning. Though it may not be the intended task of ooDSGCs, in the VME groups, using this decoding method, we found that their firing rates can be used to decode speed or contrast at higher accuracy than control, especially under weaker stimulus conditions such as low contrast and high speed (data not shown), possibly also due to enhanced response reliability.

\section{Effect of response reliability on motion encoding}

After early visual motion training, VME ooDSGCs respond to motion with higher response reliability than the control ooDSGCs under most stimulus contrasts and speeds tested (Fig. $5 D, E)$. This has a positive impact on the coding of motion directions. We showed that switching the response variability between control and VME ooDSGCs mostly switched their direction encoding capacities, demonstrating that the difference in coding between VME and control comes mostly, if not entirely, from the difference in response reliability. Consistent with this observation, VME training at adult ages has no effect on direction decoding performance (Fig. $2 \mathrm{H}$ ), and when we examined the response reliability of these ooDSGCs, no difference was found between them and those from the control group (data not shown).

Even under normal conditions, response reliability is one of the main influencing factors behind direction encoding. As we and others have noted (Nowak et al., 2011; Figs. 2A,B, 3A,B), ooDSGCs maintain a stable TC shape under many different motion conditions, such as speed, contrast, and luminance. However, the direction decoding accuracy from ooDSGC responses can differ dramatically (Figs. 2D, 3C). If we look at the response variability of ooDSGCs under different contrasts and speeds, it became apparent that there is a tight correlation between the response variability and direction decoding accuracy. This is intuitively understandable: under weaker stimulus conditions, such as low contrast or high speed, ooDSGCs respond less reliably, so their direction encoding become less reliable as well; while stronger stimulus (high contrast or medium speed) elicits more reliable response from ooDSGCs, thus more reliable direction encoding. 
Response reliability and response amplitude are tightly correlate in most circumstances, stronger response is generally more reliable than weaker response. Although we showed in our simulation that the improvement on motion encoding completely depend on enhanced response reliability, we also observed that VME ooDSGCs, in addition to enhanced response reliability, responded more strongly to some motion stimuli (Fig. $3 A$ ). This change may have an additional boosting effect on response reliability.

\section{Circuit change induced by VME training}

One surprising aspect of our findings is that the direction of training motion is irrelevant. As long as motion dominated visual environment was provided, ooDSGCs develop heightened response reliability and direction encoding capability for all directions of motion. Our mice were free-moving during VME training. Therefore, although one can say the visual environment is dominate by one direction of motion, this direction, when projected onto the retina, is not constant, it is affected by changes in head orientation and body motion. Perhaps more importantly, ooDSGCs in the mouse retina respond to all directions of motion. Their response to ND may be much weaker than to the $\mathrm{PD}$, but still significantly higher than background firing in the absence of a stimulus in most cases. Thus, all ooDSGCs are excited more strongly during VME training than without the training. These may in part explain why all ooDSGCs are affected by VME training of a single direction of motion. Furthermore, one must note that although ooDSGCs are now classified to be four distinct subtypes of RGCs based on their PDs, they arborize in the same sublaminae of the retina and receive synaptic inputs from the same group of retinal interneurons, both excitatory and inhibitory. Thus, if the VME induced circuit changes lie mainly upstream of ooDSGC, then the entire ooDSGC population would share the same effect induced by the circuit change. This may indeed be the case: ooDSGCs in the VME and control group are not significantly different in their input resistance and other electrical properties (data not shown), suggesting the changes in their visual response lie in the upstream circuit. We then showed that $\mathrm{ON}$ excitatory inputs to VME ooDSGCs are significantly more reliable than control (Fig. 6E,F), identifying that the most likely change in ooDSGC circuit to account for the improved motion encoding lie in the ON bipolar pathway.

Studies on the ON bipolar cells that innervate the ooDSGCs at different stages of the VME training will help to elucidate the exact changes induced by VME and help understand the underlying cellular and circuit mechanisms. Perhaps more interestingly, these $\mathrm{ON}$ bipolar cells may also provide inputs to other RGCs. Indeed, when we examined ON-RGCs recorded in the VME trained retinas, lowering of CV was observed (Fig. 6G,H). The effect seems to be widespread, as the average change among all ON-RGCs is large and similar to the change observed in ooDSGCs. It is worth noting that our analysis so far does not distinguish different subtype of ON-RGCs, therefore it is not yet clear whether the effect is restricted to a subset of ON bipolar pathways. Closer examination of different $\mathrm{ON}$ pathways is needed to clarify this. Moreover, VME training would keep most retinal neurons in a much more elevated activity level than control, just as ooDSGCs, so the effect we observed in ooDSGCs might not be unique. Examination of the VME effect on other RGCs are underway to determine whether training by motion may affect other RGCs sensitive to motion as well.

\section{References}

Barlow HB, Hill RM, Levick WR (1964) Retinal ganglion cells responding selectively to direction and speed of image motion in the rabbit. J Physiol 173:377-407.

Blakemore C, Cooper GF (1970) Development of the brain depends on the visual environment. Nature 228:477-478.

Borges S, Berry M (1978) The effects of dark rearing on the development of the visual cortex of the rat. J Comp Neurol 180:277-300.

Bos R, Gainer C, Feller MB (2016) Role for visual experience in the development of direction-selective circuits. Curr Biol 26:1367-1375.

Briggman KL, Helmstaedter M, Denk W (2011) Wiring specificity in the direction-selectivity circuit of the retina. Nature 471:183-188.

Chalupa LM, Rhoades RW (1978) Directional selectivity in hamster superior colliculus is modified by strobe-rearing but not by dark-rearing. Science 199:998-1001.

Chen M, Weng S, Deng Q, Xu Z, He S (2009) Physiological properties of direction-selective ganglion cells in early postnatal and adult mouse retina. J Physiol 587:819-828.

Crair MC, Gillespie DC, Stryker MP (1998) The role of visual experience in the development of columns in cat visual cortex. Science 279:566-570.

Daw NW, Wyatt HJ (1974) Raising rabbits in a moving visual environment: an attempt to modify directional sensitivity in the retina. J Physiol 240:309-330.

Demb JB (2007) Cellular mechanisms for direction selectivity in the retina. Neuron 55:179-186.

Dhande OS, Estevez ME, Quattrochi LE, El-Danaf RN, Nguyen PL, Berson DM, Huberman AD (2013) Genetic dissection of retinal inputs to brainstem nuclei controlling image stabilization. J Neurosci 33:17797-17813.

Dunn FA, Della Santina L, Parker ED, Wong RO (2013) Sensory experience shapes the development of the visual system's first synapse. Neuron 80:1159-1166.

Elstrott J, Anishchenko A, Greschner M, Sher A, Litke AM, Chichilnisky EJ, Feller MB (2008) Direction selectivity in the retina is established independent of visual experience and cholinergic retinal waves. Neuron 58:499-506.

Engert F, Tao HW, Zhang LI, Poo MM (2002) Moving visual stimuli rapidly induce direction sensitivity of developing tectal neurons. Nature 419:470-475.

Fiscella M, Franke F, Farrow K, Müller J, Roska B, da Silveira RA, Hierlemann A (2015) Visual coding with a population of direction-selective neurons. J Neurophysiol 114:2485-2499.

Franke F, Fiscella M, Sevelev M, Roska B, Hierlemann A, da Silveira RA (2016) Structures of neural correlation and how they favor coding. Neuron 89:409-422.

Hensch TK (2005) Critical period plasticity in local cortical circuits. Nat Rev Neurosci 6:877-888.

Hirsch HV, Spinelli DN (1970) Visual experience modifies distribution of horizontally and vertically oriented receptive fields in cats. Science 168:869-871.

Hooks BM, Chen C (2007) Critical periods in the visual system: changing views for a model of experience-dependent plasticity. Neuron 56:312326.

Hubel DH, Wiesel TN (1970) The period of susceptibility to the physiological effects of unilateral eye closure in kittens. J Physiol 206:419-436.

Huberman AD, Wei W, Elstrott J, Stafford BK, Feller MB, Barres BA (2009) Genetic identification of an On-Off direction-selective retinal ganglion cell subtype reveals a layer-specific subcortical map of posterior motion. Neuron 62:327-334.

Jaepel J, Hübener M, Bonhoeffer T, Rose T (2017) Lateral geniculate neurons projecting to primary visual cortex show ocular dominance plasticity in adult mice. Nat Neurosci 20:1708-1714.

Kay JN, De la Huerta I, Kim IJ, Zhang Y, Yamagata M, Chu MW, Meister M, Sanes JR (2011) Retinal ganglion cells with distinct directional preferences differ in molecular identity, structure, and central projections. J Neurosci 31:7753-7762.

Kim IJ, Zhang Y, Yamagata M, Meister M, Sanes JR (2008) Molecular identification of a retinal cell type that responds to upward motion. Nature 452:478-482.

Kim IJ, Zhang Y, Meister M, Sanes JR (2010) Laminar restriction of retinal ganglion cell dendrites and axons: subtype-specific developmental patterns revealed with transgenic markers. J Neurosci 30:1452-1462. 
Kreile AK, Bonhoeffer T, Hübener M (2011) Altered visual experience induces instructive changes of orientation preference in mouse visual cortex. J Neurosci 31:13911-13920.

Kühn NK, Gollisch T (2016) Joint encoding of object motion and motion direction in the salamander retina. J Neurosci 36:12203-12216.

Kühn NK, Gollisch T (2019) Activity correlations between direction-selective retinal ganglion cells synergistically enhance motion decoding from complex visual scenes. Neuron 101:963-976.e7.

Levelt CN, Hübener M (2012) Critical-period plasticity in the visual cortex. Annu Rev Neurosci 35:309-330.

Levine MW, Shefner JM (1977) A model for the variability of interspike intervals during sustained firing of a retinal neuron. Biophys J 19:241252.

Li Y, Van Hooser SD, Mazurek M, White LE, Fitzpatrick D (2008) Experience with moving visual stimuli drives the early development of cortical direction selectivity. Nature 456:952-956.

Liu A, Savya S, Urban NN (2016) Early odorant exposure increases the number of mitral and tufted cells associated with a single glomerulus. J Neurosci 36:11646-11653.

Melamed E, Frucht Y, Vidauri J, Uzzan A, Rosenthal J (1986) Effect of postnatal light deprivation on the ontogenesis of dopamine neurons in rat retina. Brain Res 391:280-284.

Nowak P, Dobbins AC, Gawne TJ, Grzywacz NM, Amthor FR (2011) Separability of stimulus parameter encoding by on-off directionally selective rabbit retinal ganglion cells. J Neurophysiol 105:2083-2099.

O’Hashi K, Miyashita M, Tanaka S (2007) Experience-dependent orientation plasticity in the visual cortex of rats chronically exposed to a single orientation. Neurosci Res 58:86-90.

Oyster CW, Barlow HB (1967) Direction-selective units in rabbit retina: distribution of preferred directions. Science 155:841-842.

Rivlin-Etzion M, Zhou K, Wei W, Elstrott J, Nguyen PL, Barres BA, Huberman AD, Feller MB (2011) Transgenic mice reveal unexpected diversity of on-off direction-selective retinal ganglion cell subtypes and brain structures involved in motion processing. J Neurosci 31:8760-8769.

Rivlin-Etzion M, Wei W, Feller MB (2012) Visual stimulation reverses the directional preference of direction-selective retinal ganglion cells. Neuron 76:518-525.

Rose T, Bonhoeffer T (2018) Experience-dependent plasticity in the lateral geniculate nucleus. Curr Opin Neurobiol 53:22-28.
Sabbah S, Gemmer JA, Bhatia-Lin A, Manoff G, Castro G, Siegel JK, Jeffery N, Berson DM (2017) A retinal code for motion along the gravitational and body axes. Nature 546:492-497.

Salinas E, Abbott LF (1994) Vector reconstruction from firing rates. J Comput Neurosci 1:89-107.

Sengpiel F, Stawinski P, Bonhoeffer T (1999) Influence of experience on orientation maps in cat visual cortex. Nat Neurosci 2:727-732.

Smith SL, Trachtenberg JT (2007) Experience-dependent binocular competition in the visual cortex begins at eye opening. Nat Neurosci 10:370-375.

Taylor WR, Vaney DI (2002) Diverse synaptic mechanisms generate direction selectivity in the rabbit retina. J Neurosci 22:7712-7720.

Tian N, Copenhagen DR (2001) Visual deprivation alters development of synaptic function in inner retina after eye opening. Neuron 32:439-449.

van Hateren JH (1990) Directional tuning curves, elementary movement detectors, and the estimation of the direction of visual movement. Vision Res 30:603-614.

von Trapp G, Buran BN, Sen K, Semple MN, Sanes DH (2016) A decline in response variability improves neural signal detection during auditory task performance. J Neurosci 36:11097-11106.

Wei W, Elstrott J, Feller MB (2010) Two-photon targeted recording of GFPexpressing neurons for light responses and live-cell imaging in the mouse retina. Nat Protoc 5:1347-1352.

Wei W, Hamby AM, Zhou K, Feller MB (2011) Development of asymmetric inhibition underlying direction selectivity in the retina. Nature 469:402406.

Wiesel TN, Hubel DH (1963) Single-cell responses in striate cortex of kittens deprived of vision in one eye. J Neurophysiol 26:1003-1017.

Yao X, Cafaro J, McLaughlin AJ, Postma FR, Paul DL, Awatramani G, Field GD (2018) Gap junctions contribute to differential light adaptation across direction-selective retinal ganglion cells. Neuron 100:216-228.e6.

Yonehara K, Fiscella M, Drinnenberg A, Esposti F, Trenholm S, Krol J, Franke F, Scherf BG, Kusnyerik A, Müller J, Szabo A, Jüttner J, Cordoba F, Reddy AP, Németh J, Nagy ZZ, Munier F, Hierlemann A, Roska B (2016) Congenital nystagmus gene FRMD7 is necessary for establishing a neuronal circuit asymmetry for direction selectivity. Neuron 89:177-193.

Zhang LI, Bao S, Merzenich MM (2001) Persistent and specific influences of early acoustic environments on primary auditory cortex. Nat Neurosci 4:1123-1130.

Zylberberg J, Cafaro J, Turner MH, Shea-Brown E, Rieke F (2016) Directionselective circuits shape noise to ensure a precise population code. Neuron 89:369-383. 